\title{
Analysis of clinical characteristics and efficacy of chronic myeloid leukemia onset with extreme thrombocytosis in the era of tyrosine kinase inhibitors
}

\author{
This article was published in the following Dove Press journal: \\ OncoTargets and Therapy \\ 17 July 2017 \\ Number of times this article has been viewed
}

\author{
Zhihe Liu \\ Hongqiong Fan \\ Yuying Li \\ Chunshui Liu \\ Department of Hematology, Cancer \\ Center, The First Hospital of Jilin \\ University, Changchun, People's \\ Republic of China
}

\begin{abstract}
The aim of this study was to investigate the clinical characteristics and efficacy of chronic myeloid leukemia (CML) onset with extreme thrombocytosis. A total of 121 newly diagnosed and untreated CML patients in chronic phase with complete clinical information from the First Hospital of Jilin University, from January 2010 to December 2014 were retrospectively recruited. Based on the platelet (PLT) count, 22 patients were assigned into CML with thrombocytosis (CML-T) group (PLT $>1,000 \times 10^{9} / \mathrm{L}$ ) and 65 patients were classified into CML without extreme thrombocytosis $(\mathrm{CML}-\mathrm{N})$ group (PLT $\left.\leq 1,000 \times 10^{9} / \mathrm{L}\right)$. Fifty-four point five percent of patients in the CML-T group were female, which was higher than that in the CML-N group (27.7\%) $(P=0.022)$. Except for gender, there was no significant difference for clinical information of patients between the two groups. For Sokal and Hasford scoring systems, the percentage of patients at high risk in the CML-T group were higher than those in the CML-N group, $95.5 \%$ vs $52.3 \%(P=0.000)$ and $68.2 \%$ vs $41.5 \%$ ( $P=0.031$ ), respectively; however, there was no significant difference for European Treatment and Outcome Study (EUTOS) score system between the two groups $(P=0.213)$. In terms of major molecular response (MMR) rate, the percent of patients with MMR in CML-T group was lower than that in CML-N group at 36 months after tyrosine kinase inhibitor therapy $(P=0.037)$. Up until December 2016, the median of event-free survival was 21 months in the CML-T group, however, that was not reached in the CML-N group $(P=0.027)$. The majority of CML patients with extreme thrombocytosis were females, and compared to patients in the CML-N group, the percentage of high risk patients based on the Sokal and Hasford scoring systems was higher in the CML-T group, and the median event-free survival of patients was shorter.
\end{abstract}

Keywords: BCR/ABL1, MPNs, bone marrow, EFS, chronic phase

\section{Introduction}

Chronic myeloid leukemia (CML) is the most common disease in myeloproliferative neoplasms (MPNs) disease spectrum, deriving from the transformation of a multipotent stem cell and characterized by extreme leukocytosis, increased immature granulocytes, basophilia and eosinophilia in peripheral blood (PB). The pathogenesis of CML is closely associated with the translocation of $\mathrm{t}(9 ; 22)(\mathrm{q} 34 ; \mathrm{q} 11.2)$, which results in the $B C R / A B L 1$ fusion gene. At present, the cytogenetic hallmark, Philadelphia chromosome $(\mathrm{Ph})$ and $B C R / A B L 1$ gene, is often used to distinguish CML from other MPNs.

Thrombocytosis, defined as a platelet (PLT) count $>450 \times 10^{9} / \mathrm{L}$, is very common in chronic myeloproliferative neoplasms, and it was reported that $\sim 30 \%-50 \%$ of CML patients had thrombocytosis at the time of diagnosis. But, extreme thrombocytosis,
Correspondence: Chunshui Liu Department of Hematology, Cancer Center, The First Hospital of Jilin University, No 7I Xinmin Street, Changchun City, Jilin Province, People's Republic of China Tel +86 43I 88782054 Fax +8643188783373 Email czI375602@I26.com (c)
hereby accept the Terms. Non-commercial uses of the work are permitted without any furcher permission from Dove Medical Press Limited, provided the work is properly attributed. For permission for commercial use of this work, please see paragraphs 4.2 and 5 of our Terms (https://www.dovepress.com/terms.php) 
defined as a PLT count $>1,000 \times 10^{9} / \mathrm{L}$, is rare in CML at initial diagnosis. ${ }^{1-8}$ To date, in addition to some case reports, few articles systematically investigated the clinical characteristics and efficacy of tyrosine kinase inhibitors (TKIs) in CML patients with extreme thrombocytosis. Therefore, in order to fully understand the clinical characteristics and prognosis of those CML patients with extreme thrombocytosis, we retrospectively reviewed and analyzed the clinical information and efficacy of CML patients with extreme thrombocytosis in our hospital.

\section{Materials and methods Patients}

We retrospectively reviewed the clinical information of 121 new and untreated CML patients in the First Hospital of Jilin University in China, from January 2010 to December 2014. In this study, patients who met the following criteria were excluded: 1) accelerated phase and blast phase of CML patients at disease onset; 2) poor patient compliance; and 3) patients did not have complete clinical information and follow-up data in the database. At last, $87 \mathrm{CML}$ patients were successfully recruited in this study. Allele-specific polymerase chain reaction (AS-PCR) was conducted to identify $J A K 2 V 617 F$ and $M P L W 515 \mathrm{~K}$, and reverse transcription PCR (RT-PCR) was performed to detect $B C R / A B L 1$ fusion gene. All patients were positive for $B C R / A B L 1$ and negative for $J A K 2 \mathrm{~V} 617 \mathrm{~F}$ and $M P L W 515 \mathrm{~K}$ in this study. This study was performed in accordance with the Declaration of Helsinki, and was approved by the ethics review committee of the First Hospital of Jilin University. Written informed consent was obtained from patients or their legal guardians.

\section{Sokal, Hasford and EUTOS scoring systems}

At present, Sokal, Hasford and European Treatment and Outcome Study (EUTOS) scoring systems were often used to evaluate the prognosis of CML patients by physicians; $;^{9-11}$ therefore, all 87 patients underwent the three prognostic assessments mentioned via the website (https://www.leukemia-net.org/content/leukemias/cml/ euro and sokal score/index eng.html). According to the criterion of these prognostic assessments, some patients were diagnosed as low/intermediate-risk subgroup and other patients were evaluated as high-risk subgroup.

\section{$\mathrm{PB}$ and bone marrow smear}

$\mathrm{PB}$ and bone marrow smear tests were performed at the time of initial diagnosis of all patients in this study. In PB smear, up to 200 cells were counted for each patient, while at least
500 nucleated cells need to be calculated for each patient for bone marrow smear.

\section{Treatment and the criterion of clinical efficacy}

All patients in this study initially received imatinib (400 mg/day) (Novartis, Basel, Switzerland). Clinical efficacy was verified at the particular treatment time based on the criterion of European LeukemiaNet for CML. ${ }^{12}$ It was recommended that those patients with poor treatment response or intolerance during treatment transformed to second-generation TKIs, such as nilotinib and dasatinib.

\section{Statistics}

SPSS 20.0 software was used for the statistical analysis. Clinical information and efficacy of patients between CML with thrombocytosis (CML-T) group and CML without extreme thrombocytosis (CML-N) group were compared by Student's $t$-test or the chi-square test. Event-free survival (EFS) was defined as from initial diagnosis to the first event (death from any cause, treatment failure) or last follow-up, and analysis of EFS was performed using Kaplan-Meier method, with differences compared by log-rank test. $P \leq 0.05$ was considered as statistical significance.

\section{Results \\ Clinical information}

In this study, we retrospectively collected the clinical information, such as gender, age, white blood cell (WBC), hemoglobin (HGB), PLTs and below costal margin of patients in CML-T group and CML-N group from the database in our hospital, results showed that there was no significant difference for clinical information between the two groups, except for gender and PLT (Table 1). Compared to patients in CML-N group, the rate of female patients was obviously higher in CML-T group, $54.5 \%$ vs $27.7 \%(P=0.022)$, respectively. In terms of PLT, the median of PLT was $1,398 \times 10^{9} / \mathrm{L}$ in CML-T group; however, the median of PLT in CML-N group was only $326 \times 10^{9} / \mathrm{L}$, and there was statistical difference between CML-T group and CML-N group for PLT $(P=0.000)$.

\section{$\mathrm{PB}$ and bone marrow smear}

The clinical parameters of PB smear, including the percent of eosinophils (EO\%), basophils (BA\%) and peripheral blast count $(\mathrm{PB} \%)$, were retrospectively collected in this study, and results showed that there was no significant difference between CML-T group and CML-N group, while the percent of patients with megakaryocytes $>100$ in bone marrow in 
Table I Clinical characteristics of patients in CML-T group and CML-N group

\begin{tabular}{|c|c|c|c|}
\hline & $\begin{array}{l}\text { CML-T group } \\
(n=22)\end{array}$ & $\begin{array}{l}\text { CML-N group } \\
(n=65)\end{array}$ & $P$-value \\
\hline Gender & & & 0.022 \\
\hline Male & $45.5 \%(10 / 22)$ & $72.3 \%(47 / 65)$ & \\
\hline Female & $54.5 \%(12 / 22)$ & $27.7 \%(18 / 65)$ & \\
\hline Age & & & 0.321 \\
\hline$>60 y$ & $18.2 \%(4 / 22)$ & $7.7 \%(5 / 65)$ & \\
\hline$\leq 60 y$ & $81.8 \%(18 / 22)$ & $92.3 \%(60 / 65)$ & \\
\hline WBC $\left(\times 10^{9} / L\right)$ & & & 0.730 \\
\hline Median & 106.0 & 170.0 & \\
\hline Range & $8.9-453.0$ & $2.8-474.0$ & \\
\hline HGB (g/L) & & & 0.106 \\
\hline Median & 101 & 105 & \\
\hline Range & 59-144 & $6 I-166$ & \\
\hline PLT $\left(\times 10^{9} / \mathrm{L}\right)$ & & & 0.000 \\
\hline Median & ।,398 & 326 & \\
\hline Range & $1,038-3,649$ & II 2-737 & \\
\hline Spleen (cm) & & & 0.745 \\
\hline Median & 5.2 & 5.5 & \\
\hline Range & $0-18.0$ & $0-18.0$ & \\
\hline
\end{tabular}

Note: Significant differences between the two groups groups for these factors are shown in bold.

Abbreviations: CML, chronic myeloid leukemia; CML-T, CML with thrombocytosis; CML-N, CML without extreme thrombocytosis; $y$, years; WBC, white blood cell; HGB, hemoglobin; PLT, platelet.

CML-T group was higher than that in CML-N group, 31.8\% vs $10.8 \%(P=0.047)$, respectively (Table 2$)$.

\section{Sokal, Hasford and EUTOS scores}

Based on the results of clinical information, we used three prognostic scoring systems generally used for CML to analyze patients in this study, namely, Sokal score, Hasford score and EUTOS scoring system. Results indicated that the percent of patients with high Sokal score in CML-T group was higher than that in CML-N group, $95.5 \%$ vs $52.3 \%(P=0.000)$.

Table 2 The parameters of PB and BM smear

\begin{tabular}{|c|c|c|c|}
\hline & CML-T group & CML-N group & $P$-value \\
\hline \multicolumn{3}{|l|}{$\mathrm{EO} \%$ in $\mathrm{PB}$} & 0.100 \\
\hline Median & 5.0 & 3.2 & \\
\hline Range & $0-23.0$ & $0-18.0$ & \\
\hline \multicolumn{3}{|l|}{$\mathrm{BA} \%$ in $\mathrm{PB}$} & 0.779 \\
\hline Median & 2.8 & 2.4 & \\
\hline Range & $0-13.0$ & $0-11.0$ & \\
\hline \multicolumn{3}{|c|}{ Blast\% in PB } & 0.168 \\
\hline Median & 1.2 & 0.7 & \\
\hline Range & $0-7.0$ & $0-8.0$ & \\
\hline \multicolumn{3}{|c|}{ Megakaryocytes in BM } & 0.047 \\
\hline$>100$ & 7 & 7 & \\
\hline$\leq 100$ & 15 & 58 & \\
\hline
\end{tabular}

Note: Significant differences between the two groups groups for these factors are shown in bold.

Abbreviations: $\mathrm{PB}$, peripheral blood; $\mathrm{BM}$, bone marrow; $\mathrm{CML}$, chronic myeloid leukemia; CML-T, CML with thrombocytosis; CML-N, CML without extreme thrombocytosis; EO, eosinophils; BA, basophils.
Similar to the Sokal score, the rate of patients with high Hasford score in CML-T group was also higher than that in CML-N group, $68.2 \%$ vs $41.5 \%(P=0.031)$. However, although patients with high risk EUTOS score in CML-T group were more than those in CML-N group, there was no significant difference between the two groups $(P=0.213)$ (Table 3$)$.

\section{Second-generation TKI therapy and gene mutation}

As of December 2016, 40.9\% (9/22) of patients were transforming to second-generation TKIs in CML-T group (six cases due to treatment failure and three cases because of intolerance); however, merely $18.5 \%$ (12/65) of patients in CML-N group (nine cases attributed to treatment failure and three cases owing to intolerance) $(P=0.033)$. At the same time, ABL kinase domain mutation was examined for those patients with poor treatment response, and results indicated that three patients with T315I were found in CML-T group. However, there were three patients with ABL kinase domain mutation (E255K, M244V and T315I) in CML-N group, although the rate of mutation in CML-T group was higher than that in CML-N group, $13.6 \%$ vs $4.6 \%$, respectively. There was no significant difference between CML-T group and CML-N group $(P=0.167)$.

\section{Clinical efficacy}

With regard to clinical efficacy, we mainly explored the rate of major molecular response (MMR) after TKIs treatment between CML-T group and CML-N group, and the clinical efficacy of all patients were evaluated at 3 months, 6 months, 12 months and 36 months after TKI treatment. Results demonstrated that there was no significant difference at 3 months, 6 months and 12 months after TKI treatment between the

Table 3 Sokal, Hasford and EUTOS score of patients in CML-T group and CML-N group

\begin{tabular}{|c|c|c|c|}
\hline & $\begin{array}{l}\text { CML-T group } \\
(n=22)\end{array}$ & $\begin{array}{l}\text { CML-N group } \\
(n=65)\end{array}$ & $P$-value \\
\hline Sokal score & & & 0.000 \\
\hline Low/intermediate & $4.5 \%(1 / 22)$ & $47.7 \%(31 / 65)$ & \\
\hline High & $95.5 \%(21 / 22)$ & $52.3 \%(34 / 65)$ & \\
\hline Hasford score & & & $0.03 I$ \\
\hline Low/intermediate & $31.8 \%(7 / 22)$ & $58.5 \%(38 / 65)$ & \\
\hline High & $68.2 \%(15 / 22)$ & $41.5 \%(27 / 65)$ & \\
\hline EUTOS score & & & 0.213 \\
\hline Low & $72.7 \%(16 / 22)$ & $84.6 \%(55 / 65)$ & \\
\hline High & $27.3 \%(6 / 22)$ & $15.4 \%(10 / 65)$ & \\
\hline
\end{tabular}

Note: Significant differences between the two groups groups for these factors are shown in bold.

Abbreviations: CML, chronic myeloid leukemia; CML-T, CML with thrombocytosis; CML-N, CML without extreme thrombocytosis; EUTOS, European Treatment and Outcome Study. 
Table 4 The percent of MMR in CML-T group and CML-N group

\begin{tabular}{llll}
\hline MMR & $\begin{array}{l}\text { CML-T group } \\
(\mathbf{n}=\mathbf{2 2})\end{array}$ & $\begin{array}{l}\text { CML-N group } \\
(\mathbf{n}=\mathbf{6 5})\end{array}$ & P-value \\
\hline 3 months & $2(9.1 \%)$ & $14(21.5 \%)$ & 0.325 \\
6 months & $4(18.2 \%)$ & $19(29.2 \%)$ & 0.310 \\
12 months & $6(27.3 \%)$ & $25(38.5 \%)$ & 0.344 \\
36 months & $9(40.9 \%)$ & $43(66.2 \%)$ & $\mathbf{0 . 0 3 7}$ \\
\hline
\end{tabular}

Note: Significant differences between the two groups groups for these factors are shown in bold.

Abbreviations: MMR, major molecular response; CML, chronic myeloid leukemia; CML-T, CML with thrombocytosis; CML-N, CML without extreme thrombocytosis.

two groups; but, at 36 months after TKI therapy, the rate of MMR in CML-T group was worse than that in CML-N group, $40.9 \%$ vs $66.2 \%$, respectively, and there were significant differences $(P=0.037)$ (Table 4$)$.

\section{Survival}

Up to December 2016, all patients were followed-up effectively, and the median follow-up time of all patients was 42 months (24-82 months). Of all patients, one patient died of disease progression in April 2013 in CML-T group; however, in CML-N group, one patient died in October 2016 because of suicide after allogeneic hematopoietic stem cell transplantation, and another patient died in April 2015 as a result of disease progression. The median EFS of patients in CML-T group was 21 months; however, the median EFS of patients in CML-N group was not reached $(P=0.027)$ (Figure 1).

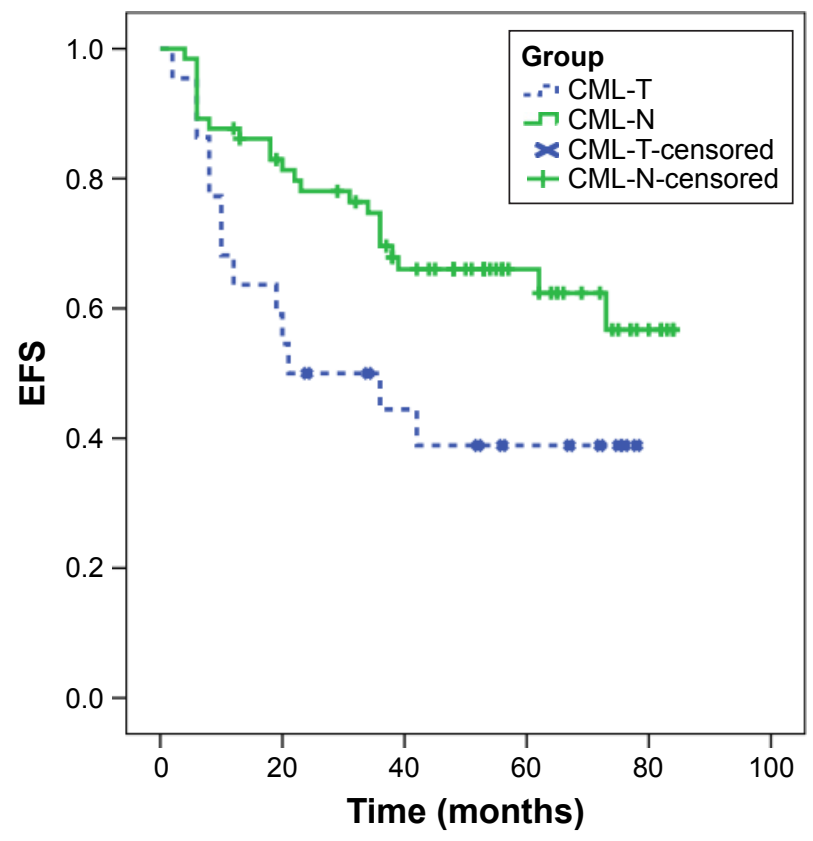

Figure I The median EFS of patients in CML-T group and CML-N group. Notes: The median EFS of patients in CML-T group was 21 months; however, the median EFS of patients in CML-N group was not reached $(P<0.05)$.

Abbreviations: EFS, event-free survival; CML, chronic myeloid leukemia; CML-T, $\mathrm{CML}$ with thrombocytosis; CML-N, CML without extreme thrombocytosis.

\section{Discussion}

In this study, unlike patients in CML-N group, the majority of patients in CML-T group were female, and the phenomenon in this study was consistent with those results previously reported. In China, there are almost $25 \mathrm{CML}$ patients with thrombocytosis reported to date; of 25 patients, 64\% were female, and the median age of disease onset was 36 years. ${ }^{7}$ Stoll et $\mathrm{al}^{13}$ and Michiels et $\mathrm{al}^{14}$ found that all CML patients with thrombocytosis were female. Morris et al ${ }^{15}$ investigated the clinical characteristics of CML patients with thrombocytosis, and results displayed that $\sim 83.3 \%$ of patients were female; therefore, we may draw a conclusion from these data previously reported, that the majority of CML patients with thrombocytosis are female.

The results of three scoring systems, Sokal, Hasford and EUTOS, were completely evaluated, and results demonstrated that there were significant differences for patients with high risk according to Sokal and Hasford scoring systems between CML-T group and CML-N group, however, there was no statistical difference between the two groups for EUTOS scoring system. It was known that Sokal and Hasford scoring systems were associated with six factors, age, spleen below costal margin, PLT count in PB, and the percent of basophils, eosinophils and myeloblasts in PB; however, EUTOS scoring system only included two factors (spleen below costal margin and basophils in PB). Therefore, we retrospectively reviewed these factors for patients in this study, and results showed that there was no significant difference for those parameters between the two groups, except for PLT count in PB. Consequently, we considered that significant differences in Sokal and Hasford scoring systems may be closely involved in extreme thrombocytosis between the two groups, and there was no doubt for no difference in EUTOS scoring system, because there was no significant difference for these two factors mentioned earlier between the two groups.

The median EFS of patients in CML-T group was much worse than those in CML-N group. In this study, there was as high as $95.5 \%$ of patients in high-risk group (Sokal scoring system) and $68.2 \%$ of patients in high-risk group (Hasford scoring system) in CML-T group, and these patients all received imatinib therapy after definite diagnosis, according to the criterion of Chinese guidelines for CML. ${ }^{16}$ At present, it was reported that the Sokal and Hasford scoring systems were significantly associated with the overall survival of CML-chronic phase patients treated with imatinib ( $P=0.014$ and 0.016 , respectively). ${ }^{17}$ Yahng et $\mathrm{al}^{18}$ also reported that all three high-risk groups (Sokal, 
Hasford and EUTOS) showed significantly lower incidence of both Complete Cytogenetic Response (CCyR) and MMR compared to low-risk groups; therefore, we considered that the poor EFS of patients in CML-T group may be involved with a higher proportion of patients with high risk in Sokal and Hasford scoring systems. For those patients with high risk in Sokal and Hasford scoring systems, it is gratifying that they could achieve better results if initially receiving second-generation TKIs therapy, rather than imatinib, ${ }^{19,20}$ but, nilotinib was not used as first-line therapy for CMLchronic phase patients until 2016 in China. ${ }^{21}$ Based on those facts mentioned earlier, we considered that clinical efficacy and survival of patients in CML-T group may be improved if they regarded nilotinib as first-line therapy after diagnosis, but the conclusion should be verified by multi-center clinical study.

Hemorrhagic and thrombotic complications are rare in CML patients; even with marked thrombocytosis, it is reported that the incidence of hemorrhagic and thrombotic events is $3 \%$ and $1 \%$, respectively. ${ }^{22}$ Sorà et al ${ }^{2}$ reviewed the behavior of CML with extreme thrombocytosis at their center, and of $100 \mathrm{CML}$ patients, only one patient developed thrombosis at diagnosis, while no patients reported a history of bleeding. Rice and Popat ${ }^{23}$ reported two cases of females who initially presented with only severe thrombocytosis and vascular events (stroke and myocardial infarction) and later developed typical features of CML. In this study, we also investigated the episodes of hemorrhagic and thrombotic events, and merely one of $85 \mathrm{CML}$ patients was detected with thrombosis at diagnosis, while no case with bleeding; therefore, we thought that elevated thrombocytosis in CML patients had no close relationship with hemorrhagic and thrombotic events.

To date, the molecular mechanisms resulting in the extreme thrombocytosis seen in CML-chronic phase patients have not been clarified. Recently, Turakhia et $\mathrm{al}^{24}$ not only reviewed the blood and bone marrow morphological findings of seven CML-T, four otherwise typical CML and one CML in blast phase but also explored the incidence of $J A K 2 \mathrm{~V} 617 \mathrm{~F}$, CALR mutation and p-STAT5 expression of those patients by RT-PCR and immunohistochemistry (IHC), and results displayed that p-STAT5 was activated in $85.7 \%(6 / 7)$ of CML-T patients. Raised thrombopoietin (TPO) and cmpl (TPO receptor) levels were observed by Karakuş et $\mathrm{al}^{25}$ in CML with thrombocytosis. Balatzenko et $\mathrm{al}^{5}$ published a case report in 2008, and results revealed that the enhanced megakaryocytopoiesis and thrombopoiesis could be due in part to the aberrant expression of the EVI1 gene. It was acknowledged that PLTs were derived from megakaryocytes in bone marrow, and interestingly, the percent of patients with megakaryocytes $>100$ in CML-T group was remarkably higher than those in CML-N group in this study. We speculate that increased megakaryocytes in CML-T group may be closely associated with the activation of certain proteins, such as STAT5 protein and EVI1 gene abnormality.

\section{Conclusion}

The vast percentage of patients in CML-T group were female, and compared to patients in CML-N group, the percentage of patients at high risk according to Sokal and Hasford scoring system was higher, and the median EFS of patients with extreme thrombocytosis was lower. We consider that patients in CML-T group might obtain better treatment response if they receive second-generation TKIs as first-line therapy.

\section{Disclosure}

The authors report no conflicts of interest in this work.

\section{References}

1. Schilling RF. Platelet millionaires. Lancet. 1980;316(8190):372-373.

2. Sorà $\mathrm{F}$, Autore $\mathrm{F}$, Chiusolo $\mathrm{P}$, et al. Extreme thrombocytosis in chronic myeloid leukemia in the era of tyrosine kinase inhibitors. Leuk Lymphoma. 2014;55(12):2958-2960.

3. Verma SP, Subbiah A, Jacob SE, Basu D. Chronic myeloid leukaemia with extreme thrombocytosis. BMJ Case Rep. 2015;2015:ii: bcr2014204564.

4. Thakral B, Saluja K, Malhotra P, Sharma RR, Marwaha N, Varma S. Therapeutic plateletpheresis in a case of symptomatic thrombocytosis in chronic myeloid leukemia. Ther Apher Dial. 2004;8(6):497-499.

5. Balatzenko G, Guenova M, Stoimenov A, Jotov G, Toshkov S. Philadelphia chromosome-positive chronic myeloid leukemia with p190BCR-ABL rearrangement, overexpression of the EVI1 gene, and extreme thrombocytosis: a case report. Cancer Genet Cytogenet. 2008;181(1):75-77.

6. Kim SY, Jeon YL, Park TS. Chronic myeloid leukemia with extreme thrombocytosis. Korean J Hematol. 2012;47(1):7.

7. Liu S, Li JJ, Wagn FY. A case of chronic myeloid leukemia onset with marked thrombocythemia and review of the literature. Mod Med J China. 2015;17(4):30-33.

8. van Kooten Niekerk PB, Roug AS, Petersen CC, et al. Chronic myeloid leukaemia presenting with isolated thrombocythaemia, a case revealing its stem cell biology. Br J Haematol. 2013;162(1):141-144.

9. Sokal JE, Cox EB, Baccarani M, et al. Prognostic discrimination in "goodrisk" chronic granulocytic leukemia. Blood. 1984;63(4):789-799.

10. Hasford J, Pfirrmann M, Hehlmann R, et al. A new prognostic score for survival of patients with chronic myeloid leukemia treated with interferon AlfaWriting Committee for the Collaborative CML Prognostic Factors Project Group. J Natl Cancer Inst. 1998;90(11):850-859.

11. Hasford J, Baccarani M, Hoffmann V, et al. Predicting complete cytogenetic response and subsequent progression-free survival in 2060 patients with CML on imatinib treatment: the EUTOS score. Blood. 2011;118(3):686-692.

12. Baccarani M, Deininger MW, Rosti G, et al. European LeukemiaNet recommendations for the management of chronic myeloid leukemia: 2013. Blood. 2013;122(6):872-884. 
13. Stoll DB, Peterson P, Exten R, et al. Clinical presentation and natural history of patients with essential thrombocythemia and the Philadelphia chromosome. Am J Hematol. 1988;27(2):77-83.

14. Michiels JJ, Berneman Z, Schroyens W, et al. Philadelphia ( $\mathrm{Ph}$ ) chromosome-positive thrombocythemia without features of chronic myeloid leukemia in peripheral blood: natural history and diagnostic differentiation from $\mathrm{Ph}$-negative essential thrombocythemia. Ann Hematol. 2004;83(8):504-512.

15. Morris CM, Fitzgerald PH, Hollings PE, et al. Essential thrombocythaemia and the Philadelphia chromosome. Br J Haematol. 1988;70(1): 13-19.

16. Chinese Society of Hematology, Chinese Medical Association. The guidelines for diagnosis and treatment of chronic myelogenous leukemia in China (2013 edition). Chin J Hematol. 2013;34(5):464-470.

17. Francis J, Dubashi B, Sundaram R, Pradhan SC, Chandrasekaran A. Influence of Sokal, Hasford, EUTOS scores and pharmacogenetic factors on the complete cytogenetic response at 1 year in chronic myeloid leukemia patients treated with imatinib. Med Oncol. 2015;32(8):1-6.

18. Yahng SA, Jang EJ, Choi SY, et al. Comparison of Sokal, Hasford and EUTOS scores in terms of long-term treatment outcome according to the risks in each prognostic model: a single center data analyzed in 255 early chronic phase chronic myeloid leukemia patients treated with frontline imatinib mesylate. Blood. 2012;120(21):2794-2794.
19. Hochhaus A, Saglio G, Hughes TP, et al. Long-term benefits and risks of frontline nilotinib vs imatinib for chronic myeloid leukemia in chronic phase: 5-year update of the randomized ENESTnd trial. Leukemia. 2016;30(5):1044-1054.

20. Hjorth-Hansen H, Stenke L, Söderlund S, et al. Dasatinib induces fast and deep responses in newly diagnosed chronic myeloid leukaemia patients in chronic phase: clinical results from a randomised phase-2 study (NordCML006). Eur J Haematol. 2015;94(3):243-250.

21. Chinese Society of Hematology, Chinese Medical Association. The guidelines for diagnosis and treatment of chronic myelogenous leukemia in China (2016 edition). Chin J Hematol. 2016;37(8):633-639.

22. Schafer AI. Bleeding and thrombosis in the myeloproliferative disorders. Blood. 1984;64(1):1-12.

23. Rice L, Popat U. Every case of essential thrombocythemia should be tested for the Philadelphia chromosome. Am J Hematol. 2005;78(1): 71-73.

24. Turakhia SK, Murugesan G, Cotta CV, Theil KS. Thrombocytosis and STAT5 activation in chronic myelogenous leukaemia are not associated with JAK2 V617F or calreticulin mutations. J Clin Pathol. 2016;69(8):713-719.

25. Karakuş S, Özcebe Oİ, Haznedaroğlu İC, et al. Circulating thrombopoietin in clonal versus reactive thrombocytosis. Hematology. 2002; $7(1): 9-12$
OncoTargets and Therapy

\section{Publish your work in this journal}

OncoTargets and Therapy is an international, peer-reviewed, open access journal focusing on the pathological basis of all cancers, potential targets for therapy and treatment protocols employed to improve the management of cancer patients. The journal also focuses on the impact of management programs and new therapeutic agents and protocols on

\section{Dovepress}

patient perspectives such as quality of life, adherence and satisfaction. The manuscript management system is completely online and includes a very quick and fair peer-review system, which is all easy to use. Visit http://www.dovepress.com/testimonials.php to read real quotes from published authors. 